\title{
A Review: Security of Data in Cloud Storage using ECC Algorithm
}

\author{
A. Harsha and Basavaraj Patil
}

\begin{abstract}
Security is the major issue in transmitting the data in present day. Security is the main focus of anxiety for Cloud Services vendors and providers. One of the procedures which the clients and users can adopt is, encrypt their data before it's stored on the cloud. The encryption process is intended to provide security services such as confidentiality, reliability and availability of the data anytime in the cloud services. Elliptic Curve Cryptography (ECC) algorithmis one of the best algorithms for the encryption of the data when compared with RSA. As ECC is having flexibility and enhanced ability with the parameters such as smaller key sizes, lower CPU time and less memory usage.
\end{abstract}

Keywords--- Cloud Computing, ECC, RSA, Security.

\section{INTRODUCTION}

$\mathrm{W}$ ITH the development of cloud technology, the existence of storing the multimedia files like text documents, photos, music files etc. on our computer has slowly reduced. Presently, the cloud service fulfills the requirements for extensive storage to accumulate all of our digital information. Peoples, who aspire their data to be hosted, purchase or rent these storage capacities from the cloud storage vendors. The cloud-data center workers virtualize the information as per the requirements of the end user and represent them as storage pools, which the end users can themselves use to accumulate and reclaim their files or data objects. Actually, the resources are accumulated over multiple servers.

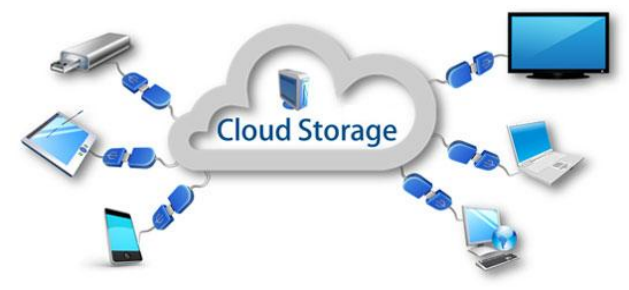

Figure 1: Cloud storage Architecture

As shown in Figure 1, cloud storage can be used from smaller computing devices to desktop computers and servers. The data accumulated in the cloud can be retrieved through a web service application interfaces or through a Web-based

A. Harsha, UG, Information science Department, SDMIT, Ujire, Karnataka.E-mail:hharsha1997@gmail.com

Basavaraj Patil, Assistant professor, Information science Department, SDMIT, Ujire, Karnataka.E-mail:bbpatilcs@gmail.com

DOI:10.9756/BIJSESC.8262 user interfaces. The cloud storage space architectures, designs a solitary virtual cloud accumulating system called storage system or cloud of clouds system. The data which is accumulated has the few following threats:

1) Since the data is stored in different locations, increases the risk of physical un-authorized access to the information.

2) As the people access the data are more, the chances of data being compromised, increases dramatically.

3) The number of communication links over the network increases. When the data travels to as local area network or storage area network, data stored on a cloud requires a Wide area network to connect them both.

4) The increase in data redundancy - Since there are multiple copies of data present across multiple servers the storage space required increases dramatically.

To secure the data on cloud, most of the users depend on join up of multiple techniques, which include:

1) Encryption -Used to encode the information. To decrypt, user needs to have the public or private key to decrypt.

2) Authentication - Validates the user is authentic or not. Verifying the user name and password it can be validated..

3) Authorization-The clients who are authorized to access the data which is stored on the cloud database.

Many organizations have multiple levels of authorization processes. For example, a frontline staff has a very limited access to information, where as the head will have all the privileges to access the files. Cloud storage approach gives a strong prospective security to the data and moreover, only the using secret word as password can be accessed to storage is not sufficient.

\section{PREVIOUS STUdY}

A. RSA

RSA is a generally used cryptography algorithm for the encryption and decryption of data. It is named after Ron Rivest, Adi Shamir, and Leonard Adleman in 1977[1]. Unlike ECC, RSA is also used to encrypt and decrypt data, with verifying digital signatures. However, its encryption and signature algorithms include a hash function, similar to ECDSA, where SHA-1 is also commonly used. The RSA is used to generate a public and private key by the order of the multiplication of two prime numbers. Therefore, rigidity of RSA and other technique to break those keys comes from solving the prime factorization problem [4]. 


\section{RSA Key Generation Process}

Input: None

Output: Public key (n, e) \&private key d.

1) Select two random prime numbers $p$ and $q$ with similar bit lengths

2) Compute $n=p q$

3) Compute $\Phi(\mathrm{n})=(\mathrm{p}-1)(\mathrm{q}-1)$

4) Compute $d$ where ed $=1 \bmod \Phi(n)$ and e is a random integer such that $\mathrm{e}<\Phi(\mathrm{n})$ and $\operatorname{gcd}(\mathrm{e}, \Phi(\mathrm{n}))=1$

5) $(\mathrm{n}, \mathrm{e})$ is the public key and d is the private key

\section{RSA Signature Generation Process}

Input: $\mathrm{d}, \mathrm{n}$, hash function $\mathrm{H}$, and message $\mathrm{m}$ Output: Signatures

1) Compute $\mathrm{s}=\mathrm{H}(\mathrm{m}) \mathrm{d}(\bmod \mathrm{n})$

2) $\mathrm{s}$ is the signature generated

\section{RSA Signature Verification Process}

Input: $\mathrm{s}, \mathrm{e}, \mathrm{n}, \mathrm{m}$, and hash function $\mathrm{H}$

Output: Accept or reject signature s

1) Compute $h=\operatorname{se}(\bmod n)$

2) If $h=H(m)$, accept the signature When comparing ECDSA to RSA, a major factor to discuss is key size. The following table shows the key lengths for RSA and ECDSA with the same level of security [10].

\section{B. $E C C$}

ECC (Elliptic Curve Cryptography) was discovered by Victor Miller (IBM) and Neil Koblitz (University of Washington) in 1985 as an alternative method for designing public-key cryptography. This method depends on elliptical curve theory. It can be used to create a quicker, smaller, \&more proficient cryptographic keys. ECC generates keys through the possessions of the elliptic curves expression as a substitute of conventional method of keys generation process.

Similar to some traditional algorithms such as RSA, ECC is also based on discrete logarithms which are much complicated to challenge at its correspondent key lengths. According to a research, ECC gives security with 164-bit key, other systems needs a 1,024 -bit key to attain. As ECC provides security with lesser computing power and battery resource usage, it is can be used for mobile appliances.

\section{ECC Key Generation Process}

Key generation is first and major step in any algorithm. It is used to produce public key and private keys. The sender will encryptor convert the input message with public key of receiver and the receiver will decode or decrypt the message with his own private key. Now, select a number' $d$ ', within the range of zero to $\mathrm{n}$. Generate the public key using the equation as follows,

$$
Q=d * P
$$

Where, $\mathrm{d}=$ random number selected (1 to $\mathrm{n}-1)$.

$\mathrm{P}$ is the point on the curve,

$\mathrm{Q}$ is the public key

$\mathrm{d}$ is the private key.

\section{ECC Signature Generation Process}

Let ' $\mathrm{m}$ ' be the data message that has to be sent. Consider ' $m$ ' has the point ' $M$ ' on the curve ' $E$ '. Randomly select ' $k$ ' from 1 to (n-1). Two cipher texts will be generated let it be $\mathrm{C} 1$ and $\mathrm{C} 2$.

$$
\mathrm{C} 1=\mathrm{k} * \mathrm{P} \text { and } \mathrm{C} 2=\mathrm{M}+(\mathrm{k} * \mathrm{P})
$$

\section{ECC Signature Verification Process}

Use the below equations we can get the original message ' $m$ ' back which was sent.

$$
\mathrm{M}=\mathrm{C} 2-\mathrm{d} * \mathrm{C} 1
$$

Where, $\mathrm{M}$ is the original message that was sent.

\section{COMPARISON OF ECC AND RSA}

Here, we compare both ECC as well as RSA algorithms, by considering the three major parameters, key size, performance and bandwidth.

\section{A. Key Size}

The first important parameter to select for the implementation of any algorithm is size of the key. It is good practice of selecting a very large key size for better security. Larger sized key means even additional expensive computation time. The key challenge is to identify the smallest sized key which would be more secure [18].

Table1: Comparison of ECC \& RSA - Key Size.

\begin{tabular}{|c|c|}
\hline ECC (in bits) & RSA (in bits) \\
\hline 106 & 512 \\
\hline 112 & 768 \\
\hline 132 & 1024 \\
\hline 160 & 2048 \\
\hline 210 & 3072 \\
\hline 283 & 7680 \\
\hline 409 & 15360 \\
\hline 571 & 21000 \\
\hline
\end{tabular}

\section{B. Performance Anxiety}

When we design any system, we need a better performance form it. For that, performance at 128-bit security levels, RSA is generally to be 10-times, slower than ECC. The performance inequality enhances considerably at 256-bit security levels, where the RSA method is 50-100times slower than ECC. ECC key generation is faster as compared to RSA's key generation process. However, this may or may not be a significant deliberation in systems that generate keys frequently. It matters for some protocols or that needs more recurrent key generation methods. Public key signature validation is generally faster with RSA compared to ECC[18].

\section{Bandwidth}

When it comes to network bandwidth, the number one concern relates to the symmetric algorithm used for message encryption and Message Authentication Coding (MAC) for 
integrity checking (this is unrelated to the choice of RSA versus ECC). Smaller embedded systems may start sessions more frequently, or the asymmetric authentication may be a larger percentage of the overall traffic and the size of the keys and signatures can make a difference. The 128-bit security level, public signatures \&keys are 6-times bigger than ECC. Private keys are 12-times larger than ECC at the 128-bit security level. The key size has lesser impact on performance, but size matters when we consider about the cost of secure storage [18].

\section{Advantages of ECC over RSA}

1) Smaller keys of ECC are as strong as longerkeys of RSA.

2) CPU utilization is very LOW.

3) Memory consumption is also very LOW.

4) Size of encrypted data is much smaller compared to RSA.

\section{Simulation}

A. Assumptions

1) Block Size: The size of block is presumed to be as follows. The same block sizes are used for both ECC and RSA which is based on the key size.

1) Encryption Size of block: ((key size / 8) - 11).

2) Decryption Size of block: (key size / 8).

2) Key Size: According to NIST (National Institute of Standards and Technology) policies ECC and RSA have equivalent key sizes with corresponding security levels.

3) Parameters: To evaluate the performance of the RSA and ECC algorithms, the following are considered for simulation:

1) Time required for Key Generation.

2) Time required Encryption.

3) Time required Decryption.

Since there was a timing difference in each of these 3 parameters, simulation was conducted repetitively for about 20 times to find the average timings.

\section{ANALYSIS OF TEST RESUlTS}

\section{A. Key Generation Time}

The times taken from both the system are not same. It can take very long time to generate the keys sometimes, even for same key size. Figure 2 [23], represents the comparison of the key generation. The time taken is almost equal in both cases, but as the key size increases RSA takes more amount of time to generate the keys and this time increases exponentially with the key size[22].

Table 2: Key Generation

\begin{tabular}{|c|c|c|c|}
\hline \multicolumn{4}{|c|}{ Key Generation } \\
\hline \multicolumn{2}{|c|}{ Key Lengths(bits) } & \multicolumn{2}{c|}{ Time(sec) } \\
\hline ECC & RSA & ECC & RSA \\
\hline 163 & 1024 & 0.08 & 0.16 \\
\hline 233 & 2240 & 0.18 & 7.47 \\
\hline 283 & 3072 & 0.27 & 9.80 \\
\hline 409 & 7680 & 0.64 & 133.90 \\
\hline 571 & 15360 & 1.44 & 679.06 \\
\hline
\end{tabular}

Figure 2 shows the comparison of time taken for key generation between RSA and ECC algorithms.

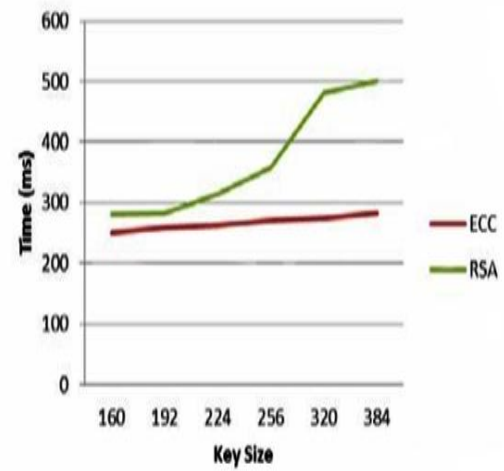

Figure 2: Comparison of ECC and RSA key Generation Time.

\section{B. Encryption/Decryption Time}

Figure 3 represents the time taken to encrypt for ECC and RSA algorithms [23].

The implementation of RSA in Java doesn't support key sizes lesser than 512 bits length. The simulation is to compare the encryption/decryption times between these algorithms with different key sizes. Considering the end results, for smaller key sizes ECC gives much faster encryption/decryption than RSA. In the analysis of tests, the results shows that, encryption using ECC is much faster when compared to RSA. The graphs indicate that the encryption time of ECC varies linearly and the encryption time of RSA increases exponentially with respect to the time.

Table 3: Signature Generation

\begin{tabular}{|c|c|c|c|}
\hline \multicolumn{4}{|c|}{ Signature Generation } \\
\hline \multicolumn{2}{|c|}{ Key Length (bits) } & \multicolumn{2}{c|}{ Time(sec) } \\
\hline ECC & RSA & ECC & RSA \\
\hline 163 & 1024 & 0.15 & 0.01 \\
\hline 233 & 2240 & 0.34 & 0.15 \\
\hline 283 & 3072 & 0.59 & 0.21 \\
\hline 409 & 7680 & 1.18 & 1.53 \\
\hline 571 & 15360 & 3.07 & 9.20 \\
\hline
\end{tabular}

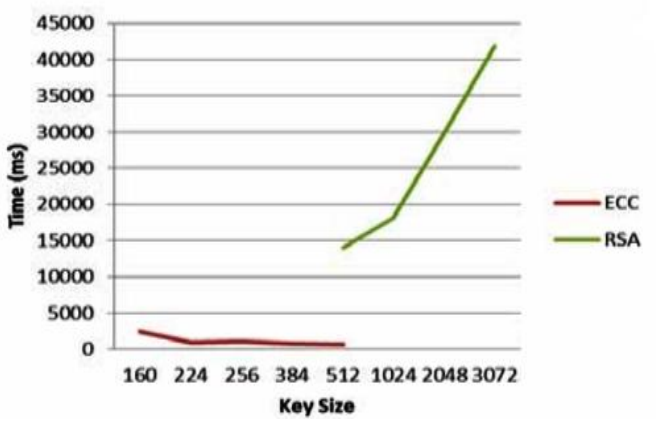

Figure 3: Comparison of Encryption Times

For both algorithms, decryption time is lesser than encryption time. But the decryption time taken by ECC is 
much high when compared with RSA even at both smaller as well as longer key lengths as show in figure4.Table 4 compares the signature verification time taken by both ECC and RSA, determines that the RSA algorithm defeats ECC by taking much less time for decryption at smaller as well as at larger key lengths.

Table 4: Signature Verification

\begin{tabular}{|c|c|c|c|}
\hline \multicolumn{4}{|c|}{ Signature Verification } \\
\hline \multicolumn{2}{|c|}{ Key Length (bits) } & \multicolumn{2}{c|}{ Time (sec) } \\
\hline ECC & RSA & ECC & RSA \\
\hline 163 & 1024 & 0.23 & 0.01 \\
\hline 233 & 2240 & 0.51 & 0.01 \\
\hline 283 & 3072 & 0.86 & 0.01 \\
\hline 409 & 7680 & 1.80 & 0.01 \\
\hline 571 & 15360 & 4.53 & 0.03 \\
\hline
\end{tabular}

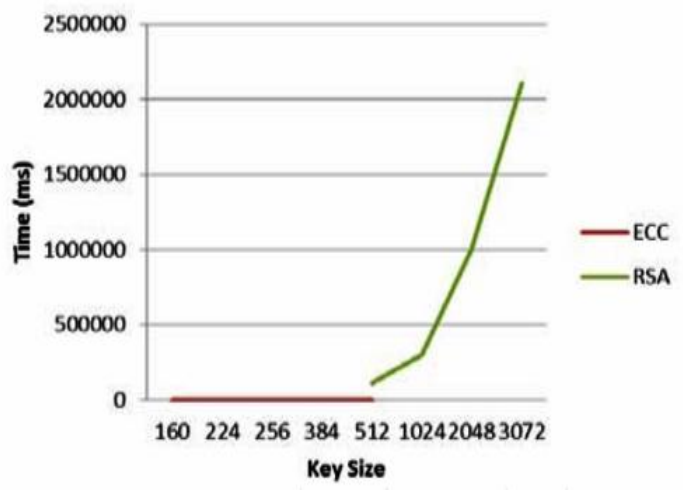

Figure 4: Comparison of decryption times

\section{CONCLUSION AND FUTURE WORK}

This paper enlightens the differences between ECC and RSA algorithms in terms of key size and time taken for encryption \& decryption. After evaluating the RSA and ECC ciphers, the ECC is less overhead to RSA. The study involved with the comparison of key size, performance analysis and bandwidth utilization. ECC algorithm has smaller key size lesser computing complexity then RSA.

\section{REFERENCE}

[1] N. Koblitz and J. Alfred Menezes, "A Survey of Public- Key Cryptosystems", In Second International Conference on Advanced Computing \& Communication Technologies (ACCT), 2012.

[2] R.S. Dhakar, A.K. Gupta and P. Sharma, "Modified RSA encryption algorithm (MREA)", In 2012 Second International Conference on Advanced Computing \& Communication Technologies, Pp. 426-429, 2012.

[3] M. Savari, M. Montazerolzohour and Y.E. Thiam, "Comparison of ECC and RSA algorithm in multipurpose smart card application”, In 2012 International Conference on Cyber Security, Cyber Warfare and Digital Forensic (CyberSec), Pp. 49-53, 2012.

[4] P.R. Vijayalakshmi and K. Bommanna Raja, "Performance Analysis of RSA and ECC in Identity-Based Authenticated New Multiparty Key Agreement Protocol", In International Conference on Computing, Communication and Applications (ICCCA), Pp. 1-5, 2012.

[5] ECC, https://en.wikipedia.org/wiki/Elliptic_curve_cryptography

[6] RSA (algorithm), http://en.wikipedia.org/wiki/RSA_(algorithm)
[7] T.M. Java "Cryptography Extension (JCE)", Reference Guide.

[8] I.Z. Berta and Z. Mann, "Implementing elliptic curve cryptography on PC and smart card", Periodica Polytechnica, Electrical Engineering, Vol. 46, No. 1, Pp. 47-73, 2002.

[9] M. Brown, D.L. Hankerson, J.L._opez and A. Menezes, "Software implementation of the NIST Elliptic curves over prime fields", In Progress in Cryptology - CT-RSA, D. Naccache, Vol. 2020, Pp. 250$265,2001$.

[10] Certicom Corp, "An elliptic curve cryptography (ECC) primer", White paper, Certicom, 2004.

[11] K. Rabah, "Implementation of Elliptic curve Diffie- Hellman and EC Encryption schemes", Information technology journal, 2005.

[12] K. Rabah, "Implementing Secure RSA Cryptosystem Using Your Own Cryptographic JCE Provider", Journal of Applied Science, Vol. 6, No. 3, Pp. 482-510, 2006.

[13] M.J.B. Robshaw and Y.L. Yin, "Elliptic Curve Cryptosystems", 1997.

[14] W. Stallings, "Cryptography and Network Security: Principles and Practice, 3rd edition", Prentice Hall, New Jersey, 2003.

[15] W. Trappe and L.C. Washington, "Introduction to Cryptography with Coding Theory", Prentice Hall, New Jersey, 2002.

[16] N. Weil, "U.S. govt.'s encryption standard cracked in record time", Network World, 1998,

[17] M. Amara, Lab. LAGA, Univ. Paris-8, St. Denis, France; Siad, A. "Elliptic Curve Cryptography and its applications".

[18] A.M. Fiskiran, Dept. of Electronics Engg. Princeton Univ., NJ, USA, Lee, R.B, "Workload characterization of elliptic curve cryptography and other network security algorithms for constrained environments",.

[19] S. Maria Celestin Vigila and K. Muneeswaran, "Implementation of Text based Cryptosystem using Elliptic Curve Cryptography".

[20] Atmel-8951A-CryptoAuth-RSA-ECC-Comparison-Embedded SystemsWhitePaper_07, 2015.

[21] S. Wang and G. Liu, "File encryption and decryption system based on RSA algorithm", Computational and Information Sciences (ICCIS), 2011.

[22] R. Gharshi and Suresha, "International Journal of Science and Research (IJSR)", Vol. 2, No. 7, 2013.

[23] N. Jansma and B. Arrendondo, "Performance Comparison of Elliptic Curve and RSA Digital Signatures", 2004.

\section{AUTHOR'S PROFILE}

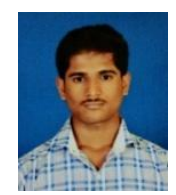

A. Harsha, is anUnder-graduate student of Shri Dharmastala Manjunateshwara Institute of Technology, Ujire. He is currently pursuing Bachelor of Engineering (ISE) under Visvesvaraya Technological University, Belagavi, Karnataka.

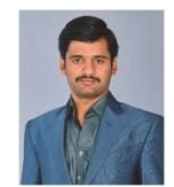

Basavaraj Patilis, working as Asst. Professor in the Department of ISE, Shri Dharmasthala Manjunatheshwara Institute of Technology Ujire, Karnataka, India. He is pursuing Ph.D at VTU, Belagavi. He received B.Eand M. Tech. degree in Computer Science \& Engineering Visvesvaraya Technological University, Belagavi in 2010 and 2013 respectively. He has published 8 research papers in National and International Journals and Conferences. He is with 4 years of teaching \& research experience. His areas of interests are Wireless Network, Image Processing and Computer Vision. 